\title{
O CURSO DE ENGENHARIA DE MINAS DA UNIVERSIDADE FEDERAL DO SUL E SUDESTE DO PARÁ: CARACTERÍSTICAS E DESAFIOS
}

\author{
Alaine Moreira Imbelloni - alaineimbelloni7@gmail.com \\ Universidade Federal do Sul e Sudeste do Pará \\ Faculdade de Engenharia de Minas e Meio Ambiente \\ Campus II, Folha 17, Quadra 04, Lote especial - Nova Marabá \\ 68507080 - Marabá - Pará \\ Denilson da Silva Costa-denilson@unifesspa.edu.br \\ Universidade Federal do Sul e Sudeste do Pará \\ Faculdade de Engenharia de Minas e Meio Ambiente \\ Campus II, Folha 17, Quadra 04, Lote especial - Nova Marabá \\ 68507080 - Marabá - Pará*
}

Carlos Alberto Pereira - pereira@demin.ufop.br

Universidade Federal de Ouro Preto

Campus Universitário, Departamento de Engenharia de Minas, Morro do Cruzeiro 35400000 - Ouro Preto - Minas Gerais.

Resumo: O ensino nos cursos de Engenharia de Minas tem sido cada vez mais desafiador para os professores e alunos, demandando mais inovações na metodologia de ensino e na utilização de recursos tecnológicos dentro e fora da sala de aula. Neste trabalho, foi realizada uma pesquisa a respeito da história do curso de Engenharia de Minas da Universidade Federal do Sul e Sudeste do Pará e suas principais características. Considerando o periodo de 2004 a 2015, foram coletadas no sistema de gestão da faculdade informações sobre o número de egressos (homens e mulheres), de evadidos e de mulheres formadas. Foi possivel perceber que, no periodo analisado, 37\% dos alunos formados na faculdade de Engenharia de Minas são mulheres, sendo um número expressivo, mas não igualitário. Observou-se também um aumento da evasão no curso nos últimos anos, o que demanda uma série de medidas corretivas para combater esse problema. Várias mudanças externas e internas à universidade podem estar relacionadas a esse comportamento, como variações no mercado de trabalho e condição financeira dos alunos. Mudanças nas metodologias de ensino, bolsas de iniciação cientifica e uma aproximação da prática com a teoria podem estimular os graduandos a vencerem essas dificuldades. Além disso, foi observado que apenas 30,83\% da carga horária do curso é destinada a aspectos especificos de engenharia de minas. Porém, alguns cursos de engenharia vêm aumentando a proporção dessas disciplinas especificas a fim de estimular os graduandos.

Palavras-chave: Engenharia de Minas. Educação. Evasão.

\section{INTRODUÇÃO}

O curso de graduação em Engenharia de Minas foi iniciado no segundo período letivo de 2004 pela Universidade Federal do Pará (UFPA). Em 2013, devido à Lei Federal 12.824 (2013), o Campus Marabá, onde funcionava o curso de Engenharia de Minas, foi desmembrado da UFPA dando origem à Universidade Federal do Sul e Sudeste do Pará (Unifesspa). 
Desde então, o curso de Engenharia de Minas, agora da Unifesspa, tem sido pioneiro na região Norte do País, oportunizado para pessoas da região a formação profissional nas áreas de pesquisa, planejamento, lavra e tratamento de minérios. Além disso, a localização privilegiada, no município de Marabá, região conhecida pela Província de Carajás, onde existem grandes projetos de Mineração, propicia a formação de engenheiros de minas na região para dar suporte ao processo de expansão dos projetos que são significativos no Estado do Pará, assim como fornecer mão de obra especializada aos novos planos.

O ensino nos cursos de Engenharia de Minas tem sido cada vez mais desafiador para os professores e para os alunos, demandando mais inovações na metodologia de ensino e na utilização de recursos tecnológicos dentro e fora da sala de aula. Para Oliveira e Pinto (2006), as mudanças no cenário global e a incorporação de novas tecnologias à produção exigem engenheiros que exerçam o seu trabalho de forma multidisciplinar, fatos que demandam remodelações curriculares dos cursos de engenharia para a formação de profissionais críticos, empreendedores, criativos e capazes de solucionar problemas.

De acordo com Borges e Almeida (2013), a educação de qualidade na graduação em engenharia é fundamental para a política estratégica de desenvolvimento social e econômico do país, que também deve priorizar ciência, tecnologia e inovação. Assim, os currículos e projetos pedagógicos dos cursos de graduação da área devem ser modernizados e a formação de mestres e doutores em Educação em Engenharia deve ser incentivada.

Lima, Oliveira e Oliveira (2012) destacam que a ausência de bons laboratórios, a falta de interesse por parte dos alunos, a dificuldade no momento de repassar a teoria para a prática são alguns dos desafios encontrados pelos professores para o ensino de engenharia. Por isso, é necessário investir tanto nas universidades como em projetos e em ações que diminuam o índice de evasão e viabilizem uma formação profissional de qualidade.

Entre os desafios para ensino superior no Brasil está a evasão, evidente em muitos cursos de engenharia. Segundo o trabalho de Prestes, Menezes e Rocha (s.d.), as principais causas do abandono dos cursos na Universidade Federal do Ceará (UFC), no campus de Crateús, foram a insatisfação com o curso, as dificuldades socioeconômicas dos alunos e alguns aspectos relacionados ao currículo do curso adicionados à escolha equivocada da graduação. Os autores destacaram que de 54 alunos que evadiram os cursos entre 2018 e 2019, muitos deles eram egressos de escolas públicas (89\%) e boa parte da Engenharia de Minas (27,8\%).

Este trabalho teve como objetivo fazer um levantamento sobre a evasão de alunos na faculdade de Engenharia de Minas da Unifesspa, assim como verificar a participação das mulheres no curso, considerando a necessidade de promover e estimular a equidade de gênero dentro da engenharia.

\section{METODOLOGIA}

Inicialmente, foi realizada uma pesquisa da literatura a respeito da história do curso na instituição, bem como as suas principais características. Em seguida, considerando o período de 2004 a 2015, foi realizada uma pesquisa no Sistema Integrado de Gestão de Atividades Acadêmicas da Unifesspa (SIGAA), onde foram coletadas as informações: número de formandos, levantamento dos números de evasões na faculdade de Engenharia de Minas e o número de mulheres formadas no curso.

Vale ressaltar que o período escolhido para estudo corresponde a todas as turmas de Engenharia de Minas que concluíram o curso até julho de 2020 pela Unifesspa. 


\section{COBENGE (C) COBENGE 2020

Figura 2 - Gráfico da proporção de evasões na faculdade de Engenharia de Minas da Unifesspa, no período de 200 a 2015

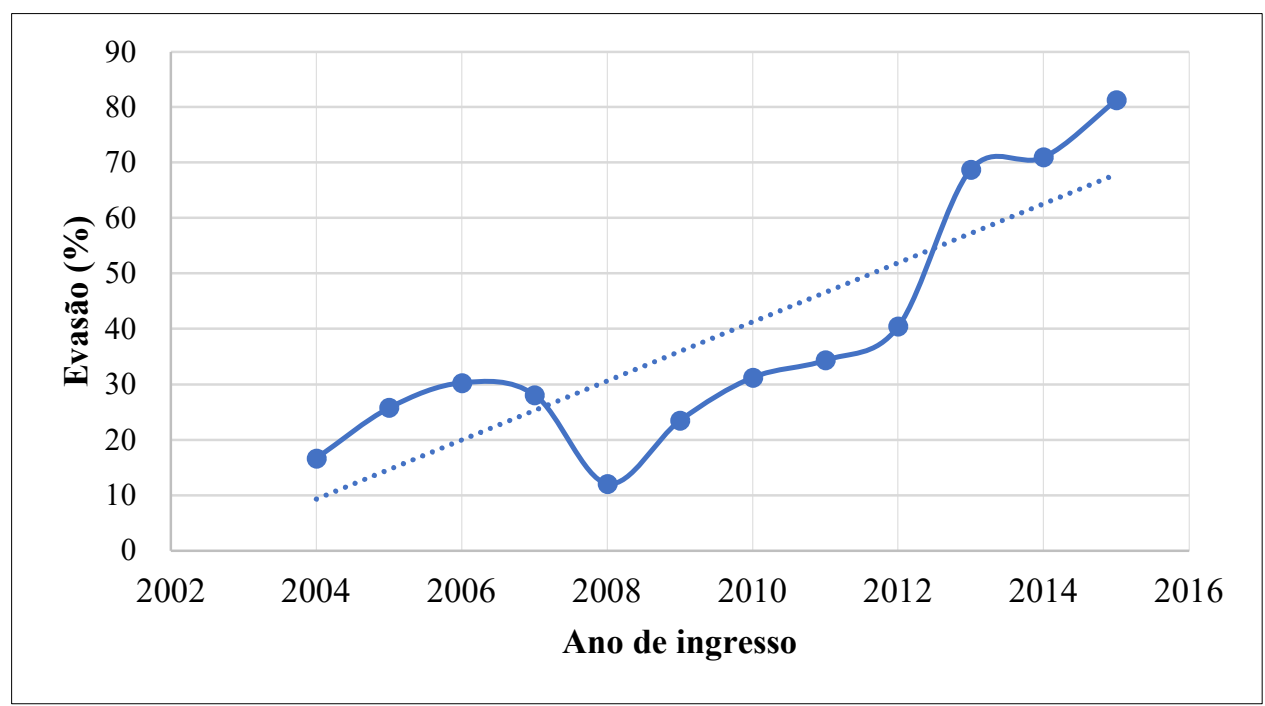

Fonte: Autores.

De acordo com o trabalho de Alvarenga, Rocha e Pereira (2011), no período de 2004 a 2011, o mercado de trabalho aumentou o número de vagas para engenheiros de minas. Analisando o gráfico da Figura 1, é possível notar que a evasão foi menor nas turmas que ingressaram nesse período, mostrando que a empregabilidade e as oscilações no mercado podem influenciar também a motivação dos alunos a permanecerem no curso.

A evasão é uma preocupação da engenharia no Brasil, no entanto alguns pontos são observados em grande parte das universidades e faculdades:

a) falta de gerenciamento dos números de evasão e retenção por parte das pró-reitorias de graduação, colegiados, departamentos e professores;

b) falta de um critério que leve em conta a produção científica do docente e a sua preparação para o cargo presidente de colegiado, pois observa-se a presença de docentes sem conhecimento das normas da Universidade ocupando esse cargo;

c) professores despreparados para receber o aluno no primeiro período.

Rios et al. (2003) apontavam esses itens e sugeriam um trabalho de orientação acadêmica que, segundo Fonseca et al. (2019), é efetivo.

Outra característica importante observada no período avaliado é o número de mulheres formadas na faculdade de Engenharia de Minas (Figuras 3 e 4). 
Figura 3 - Gráfico da proporção de mulheres em cada turma de Engenharia de Minas da Unifesspa, no período de 2004 a 2015

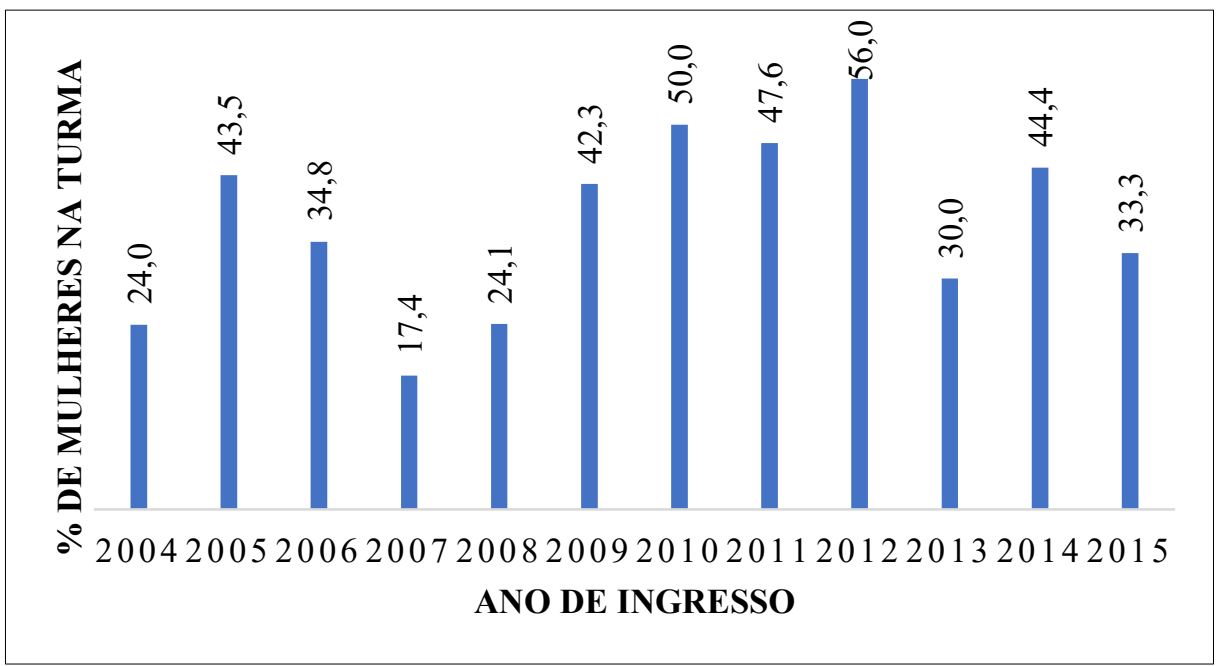

Fonte: Autores.

Figura 4 - Gráfico do número de mulheres e homens formados no curso de Engenharia de Minas da Unifesspa, no período de 2004 a 2015

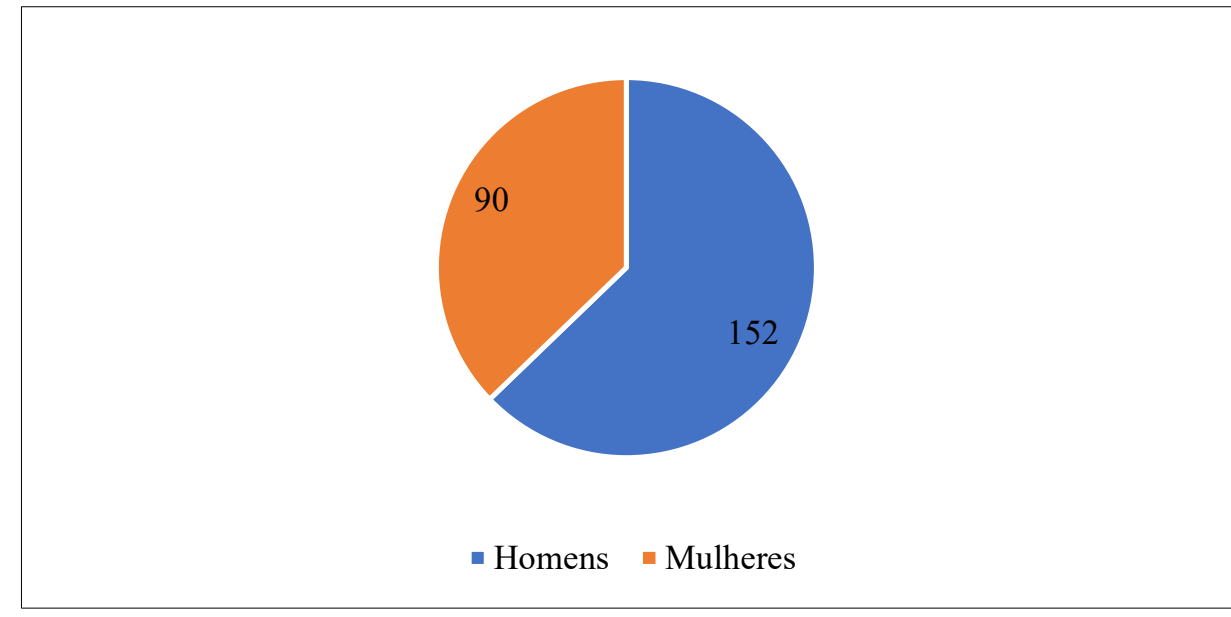

Fonte: Autores.

A partir dos gráficos nota-se que dos 242 alunos formados no curso de Engenharia de Minas da Unifesspa $37 \%$ são mulheres. É um valor expressivo, mas ainda não igualitário, 
considerando o período avaliado. Mesmo que ainda haja um número reduzido de mulheres na área tecnológica, a presença de mulheres na engenharia vem aumentando e sendo estimulada, dentro e fora da universidade. Bahia e Laudares (2011) pesquisaram sobre a presença feminina na engenharia da Pontifícia Universidade Católica de Minas Gerais (PUC) e da Universidade Federal de Minas Gerais (UFMG), no período de 2004 a 2009, e observaram que em média $28 \%$ dos inscritos nos vestibulares para Engenharia de Minas eram mulheres. Gontijo, Stopa e Pereira (2012) pesquisaram sobre a Engenharia de Minas de Ouro Preto e mostraram a evolução da presença da mulher no curso, chegando a 40\% dos formandos e, ao longo do período 1995 2011, as mulheres mostraram coeficientes de rendimento superiores aos dos homens.

O Centro Nacional de Desenvolvimento Científico e Tecnológico (CNPq) lançou editais em 2019, disponibilizando bolsas BIC-Júnior especificamente para estudantes do sexo feminino, visando à maior participação da mulher na engenharia. Analisando a presença das mulheres na docência da Engenharia de Minas, na UFOP elas representam 30\%. Na Unifesspa, por sua vez, essa proporção é similar, sendo $28 \%$ dos professores do sexo feminino. Algumas mineradoras, como Vale e Nexa Resource (antiga Votorantim Metais), têm como meta alcançar $50 \%$ de mulheres em seu quadro de engenheiros.

$\mathrm{Na}$ Tabela 1 foi feita uma comparação entre a desistência do curso considerando o gênero dos alunos. Nota-se que não há um padrão evidente de comportamento, pois o número de alunos que desistiram é bastante variável para cada ano e para cada gênero.

Tabela 1 - Porcentagem de desistência do curso de Engenharia de Minas da Unifesspe segundo os gêneros masculino (HE) e feminino (ME) no período de 2004 - 2015

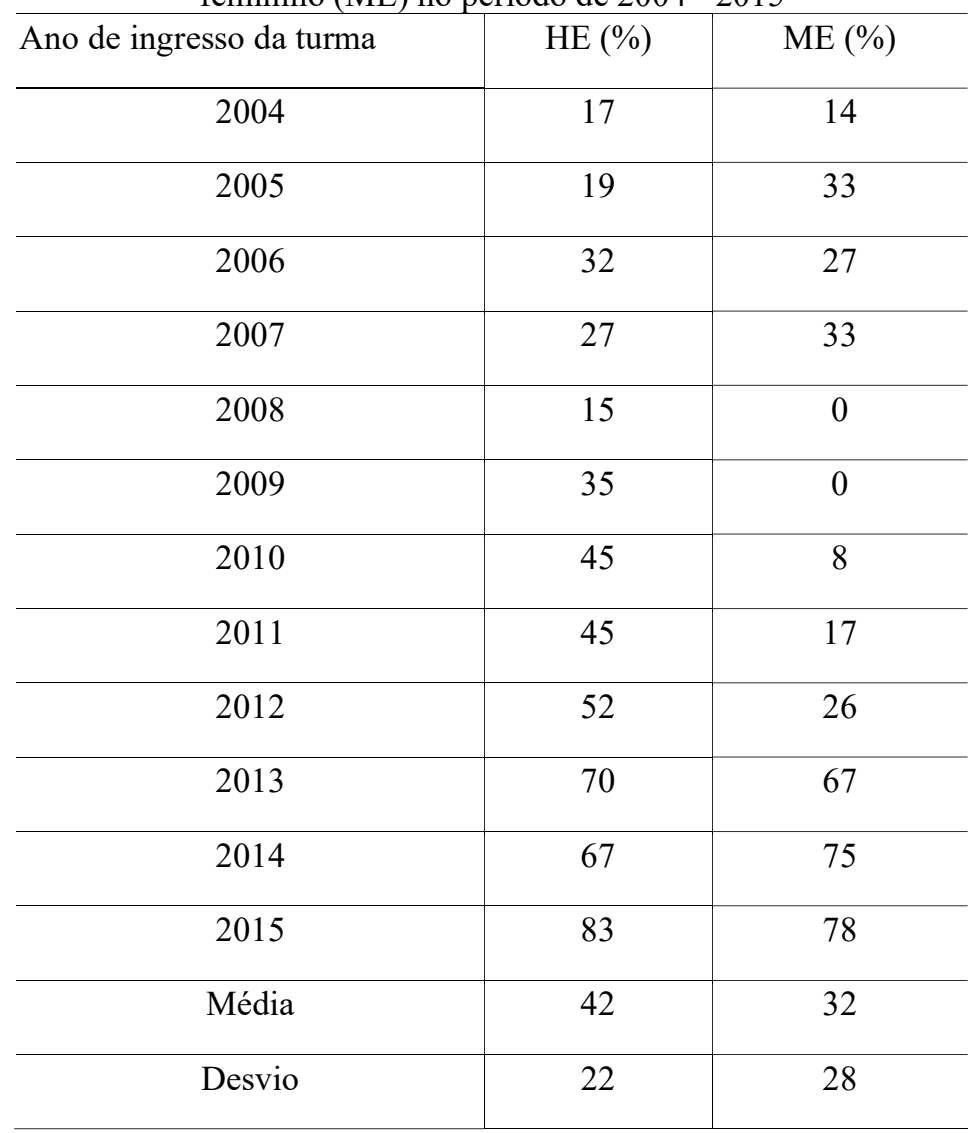


Nas últimas décadas, a quantidade de mulheres na engenharia vem aumentando, sendo por isso criado o Dia Internacional das Mulheres na Engenharia, dia 23 de junho, pela Women's Engineering Society (WES), do Reino Unido. Essa comemoração anual visa fortalecer o espaço que as mulheres vêm ganhando na profissão, em meio a tanta superação e desafios (Instituto de Engenharia, 2018). Segundo o Conselho Federal de Engenharia e Agronomia, a participação das mulheres na engenharia vem aumentando, visto que o número de engenheiras registradas anualmente no sistema teve um crescimento de $42 \%$ entre 2016 e 2018 (Conselho Regional de Engenharia e Agronomia de Alagoas, 2019).

Assim, a equidade de gênero tem sido uma discussão importante tanto no contexto profissional como no acadêmico. Estimular a participação das mulheres na engenharia, de maneira geral, tem sido um desafio em busca de uma engenharia mais igualitária. Esse desafio, entretanto, não é o único, como veremos a seguir.

\subsection{Desafios e inovações}

O ensino na engenharia tem se tornado discussão por parte da comunidade acadêmica. Isso por que, segundo Oliveira e Pinto (2006), o perfil do engenheiro no Brasil deve apresentar um conjunto de habilidades e competências para:

a) apropriar-se de novos conhecimentos e para registrar e expressar ideias de forma autônoma e independente;

b) acompanhar e contribuir para o desenvolvimento científico e tecnológico;

c) desenvolver soluções originais e criativas para os problemas de projetos, de produção e de administração;

d) trabalhar em equipe e coordenar grupos multidisciplinares;

e) conceber, projetar, executar e gerir empreendimentos de engenharia;

f) compreender e intervir na sociedade com repercussões éticas, ambientais e políticas do seu trabalho.

Além disso, os docentes de engenharia não precisam apenas dominar o conhecimento técnico e científico ou o funcionamento dos meios disponíveis para lecionar. É necessário que o ele utilize métodos e técnicas didáticas estruturados, de forma a contribuir para a formação de profissionais autodidatas, capazes de atender às demandas sociais.

Quanto aos métodos inovadores de ensino, o trabalho de Sanches, Bergerman e Roveri (2017) avaliou os cursos de Engenharia de Minas do Brasil e mostrou que, em geral, as faculdades que usam métodos inovadores nas aulas, como PBL (Project Based Learning) ou projetos em grupos, são as que possuem cursos mais recentes e disciplinas mais específicas. Ainda, estão sendo implantadas disciplinas profissionalizantes a distância.

Para Gontijo, Stopa e Pereira (2012), a evasão no curso pode ser diminuída através de ações como:

a) criar e disponibilizar um manual do aluno para que esse tenha conhecimento das oportunidades e regência da instituição;

b) aproximar o curso básico do prático, mostrando a aplicação prática das disciplinas básicas da engenharia;

c) disponibilizar tutoria ou orientação para todos alunos com dificuldades;

d) melhorar a participação de professores no oferecimento de oportunidades de trabalho na iniciação científica, extensão e ensino. 


\section{CONSIDERAÇÕES FINAIS}

Sem dúvida a educação no Brasil tem uma série de desafios, o que torna a discussão muito necessária ao desenvolvimento de políticas e medidas para estimular o aprendizado em sala de aula, tornando os cursos de engenharia mais atrativos aos estudantes.

O curso de Engenharia de Minas da Unifesspa tem apresentado um aumento da evasão de alunos nos últimos anos, o que demanda uma série de medidas corretivas para combater esse problema. Várias mudanças externas e internas à universidade podem estar relacionadas a esse comportamento: variações no mercado de trabalho, condição financeira dos alunos, quantidade de professores bem preparados, assim como a ausência de trabalhos de tutoria e eventos que possam constantemente motivar e instruir os discentes a respeito da carreira. Mudanças nas metodologias de ensino, bolsas de iniciação científica e uma aproximação da prática com a teoria também podem estimular os alunos a vencerem as suas dificuldades.

Além disso, estimular o protagonismo das mulheres na academia tem sido uma das medidas principais para a engenharia se tornar igualitária. Considerando as últimas turmas concluintes do curso de Engenharia de Minas, nota-se que a participação feminina tem sido expressiva.

Por fim, uma característica do curso que chamou a atenção foi que apenas 30,83\% da carga horária do curso são destinadas à parte específica de Engenharia de Minas. No entanto, alguns cursos de engenharia têm estimulado os alunos aumentando a proporção de disciplinas específicas, que em média corresponde a $42 \%$ da carga horária do curso.

\section{Agradecimentos \\ A Pró-reitoria de Graduação da UNIFESSPA e ao CNPq.}

\section{REFERÊNCIAS}

AlVARENGA, L. A. L.; ROCHA, G. M.; PEREIRA, C. A. Diagnóstico do Curso de Engenharia de Minas da Universidade Federal de Ouro Preto. In: XXXIX Congresso Brasileiro de Ensino de Engenharia, 2011, Blumenau. Anais. Blumenau, 2011.

BAHIA, M. M.; LAUDARES, J. B. A Engenharia e a Inserção Feminina. In.: Seminário Internacional Fazendo Gênero 10, 2013, Florianópolis. Anais Eletrônicos. Florianópolis, 2013.

BORGES, M. N.; ALMEIDA, N. N. Perspectivas para Engenharia Nacional - Desafios e Oportunidades. Revista de Ensino de Engenharia. v. 32, n. 3, 2013. Disponível em: http://revista.educacao.ws/revista/index.php/abenge/article/viewFile/238/170. Acesso em: 07 jul. 2020.

BRASIL. Lei $\mathbf{n}^{\mathbf{0}}$ 12.824. Criação da Universidade do Sul e Sudeste do Pará - UNIFSSPA, por desmembramento da Universidade Federal do Pará - UFPA, e dá outras providências. Brasília, 5 de junho de 2013. Disponível em: https://legislacao.presidencia.gov.br/atos/?tipo $=$ LEI\&numero $=12824 \&$ ano $=2013 \&$ ato $=4 \mathrm{c} 8 \mathrm{IT}$ TU50MVpWT623. Acesso em: 12 jul. 2020. 
BRASIL. Ministério da Educação. [homepage on the internet]. Dados do e-Mec sobre o ensino superior.

Disponível:https://emec. mec. gov. br/emec/consultacadastro/detalhamento/d96957f455f6405d14c6542552b0f6eb/MTg0NDA=/c1b85ea4d70 4f246bcced664fdaeddb6/RU5HRU5IQVJJQSBERSBNSU5BUyBFIE1FSU8gQU1CSUVOVEU= Acesso em: 06 jul. 2020.

CONSELHO REGIONAL DE ENGENHARIA E AGRONOMIA DE ALAGOAS. Dispara o número de mulheres engenheiras registradas no Brasil, 2019. Disponível em: http://www.creaal.org.br/2019/03/dispara-numero-de-mulheres-engenheiras-no-

brasil/\#: : :text=\%E2\%80\%9CDados $\% 20 \mathrm{do} \% 20$ Conselho $\% 20 \mathrm{Federal} \% 20 \mathrm{de}, \% \mathrm{E} 2 \% 80 \% 93 \% 2$ 0um\%20crescimento\%20de\%2042\%25. Acesso em: 15 jul. 2020.

FONSECA, A. M. V. et al. c. In.: XXVIII Encontro Nacional de Tratamento de Minérios e Metalurgia Extrativa. 2019, Belo Horizonte. Anais. Belo Horizonte, 2019.

GONTIJO, G. M.; STOPA, I. S.; PEREIRA, C. A. Evasão no Curso de Engenharia de Minas. In: XL Congresso Brasileiro de Ensino de Engenharia. Belém: Abenge, 2012. v. 1. p. 38-49.

INSTITUTO DE ENGENHARIA. Dia internacional das mulheres na engenharia. Disponível em: https://www.institutodeengenharia.org.br/site/2018/06/23/dia-internacionaldas-mulheres-na-engenharia/. Acesso em: 15 jul. 2020.

LIMA, H. F. D; OLIVEIRA, N. M. de; OLIVEIRA, N. M. De. Análise da Graduação no Ensino de Engenharia no Brasil - Análise de Dados da Universidade Federal de Campina Grande (UFCG). In: XL Congresso Brasileiro de Ensino de Engenharia, 2012, Belém. Anais. Belém, 2012.

OLIVEIRA, V. F. de; PINTO, D. P. Educação em Engenharia como Área do Conhecimento. In: XXXIV Congresso Brasileiro de Ensino de Engenharia, 2006, Passo Fundo. Anais. Passo Fundo: Ed. Universidade de Passo Fundo, 2016.

PRESTES, T. K. A; MENEZES, N. V. S; ROCHA, D. B. Evasão no ensino superior na Universidade Federal do Ceará - Campus de Crateús. In: VI Congresso Nacional de Educação - CONEDU, 2019, Fortaleza. Anais. Fortaleza, 2019.

RIOS, J. R. T; SANTOS, A. P.; LIMA, L. B. Evasão e Retenção na Escola de Minas da UFOP: A Perspectiva dos Colegiados de Cursos. In: XXXI Congresso Brasileiro de Ensino de Engenharia, 2003, Rio de Janeiro. Anais. Rio de Janeiro, 2003.

SANCHES, J. G.; BERGERMAN, M. G.; ROVERI, C. D. Uma Realização de um Comparativo com Cursos de Outros Países com Grande Produção Mineral. $18^{\circ}$ Simpósio de Mineração, 2017, São Paulo. Anais dos Seminários de Redução, Minério de Ferro e Aglomeração. São Paulo, 2017. 


\title{
THE MINING ENGINEERING COURSE OF THE UNIVERSIDADE FEDERAL DO SUL E SUDESTE DO PARÁ: CHARACTERISTICS AND CHALLENGES
}

\begin{abstract}
Teaching in mine engineering courses has been increasingly challenging for teachers and students, demanding more innovations in teaching methodology and in the use of technological resource. In this paper, a research was carried out regarding the history of the mining engineering course at the Universidade Federal do Sul e Sudeste do Pará, and its main characteristics. Considering the period from 2004 to 2015, information such as the number of graduates, survey of the number of dropouts and the number of women trained were collected in the college management system. It was possible to notice that there was an increase in dropout in the course. In addition, 37\% of the students graduated from the mining engineering college are women, which is an expressive value, but not equal. The increase in student dropout in recent years requires a series of corrective measures to combat this problem. Several changes external and internal to the university may be related to this behavior, such as variations in the job market and the financial condition of students. Changes in teaching methodologies, scholarships for scientific initiation and an approximation of practice with theory can encourage students to overcome their difficulties. In addition, it was observed that $30.83 \%$ of the course load is allocated to the specific part of mine engineering. However, some engineering courses have stimulated students by increasing the proportion of these specific disciplines.
\end{abstract}

Keywords: Mining Engineering, higher education, challenges. 